39. E. V. Dushechkina. Russkaya yolka: Istoriya. Mifologiya. Literatura [Russian Christmas tree: History. Mythology. Literature]. Norint, Saint Petersburg (2002).

40. A. P. Vasilevich, S. N. Kuznetsova, S. S. Mishchenko. Cvet i nazvaniya cveta $\mathrm{v}$ russkom yazyke [Color and color names in Russian]. KomKniga, Moscow (2005).

DOI 10.15826/B978-5-7996-3081-2.18

\title{
Resolving Structural Ambiguity in Language Processing: A Systematic Review
}

\author{
Darzhinova Liubov \\ The Education University of Hong Kong, N.T., Hong Kong \\ liubov@s.eduhk.hk
}

\begin{abstract}
This paper addresses these research questions: (1) What are the main ideas presented in the published articles (2005-2020) on structural ambiguity resolution in language processing? (2) What are the main venues for unveiling research on structural ambiguity resolution in language processing? For that, a systematic review is performed, which reports on the eight most relevant studies. It is found the investigations into the topic of interest are conducted across multidisciplinary areas and primarily in the European institutions and the US. This research is circulated in journals, which are peer-reviewed and indexed by Scopus, Web of Science, and other databases. The other major finding is that psychophysical tests are more popular in the field, and reasons for that are explained. The polarity of results on syntactic disambiguation leaves room for much to be discovered.
\end{abstract}

Keywords: written language processing, parsers, structural ambiguity resolution, systematic review

\section{Introduction}

Multidisciplinary studies have constantly been tackling the peculiarities of human languages. Those investigations have been repeatedly conducted across genetics, neurology, philosophy, linguistics, and many more research fields. Over the last few decades, there has been a considerable attention 
to research on language processing at the sentence level, which is an integral and important part of general language processing [e.g., Kaan, 2014; Lim and Christianson, 2013].

What do we already know about written language processing? In brief, as we process certain written materials, e.g., short sentences or full texts, we perform the so-called saccadic movements across words. Around two times in the course of reading some short length text, we return to what is previously read to ensure it is read accurately; and these returns are named as regressions. Once our eyes are not moving all the way through certain parts of that written material in the process of meaning extraction, these stops are called fixations [Dussias, 2010].

But how rapid do we process sentences, and how precise are we in parsing? Such a broad question has been addressed in different investigations. Among them is the bilingual study of written language processing at the sentence level via the fixed-rate window procedure [Darzhinova, 2019]. The Russian-English speaking participants were instructed to read English and Russian syntactically and semantically correct/incorrect structures. Overall, the study argues that semantic and syntactic processing in both first language (L1) and second language (L2) sentence parsing does not drastically differ from each other in terms of reaction times. Qualitative data of the study indicates that semantic processing is considerably more precise than syntactic, i.e. there are more accuracies in semantic plausibility judgements than in syntactic.

Intricate by their nature, world languages remain an extremely important tool for our communication and raise many more inquiries and concerns within their usage and comprehension. It is a matter of fact that efficacious communication between parties is dependent on many aspects, among which unambiguity comes at the onset. Nevertheless, world languages are representative of the so-called structural ambiguity, which develops once the structure of a certain linguistic stimuli suggests the prospect of a multiple interpretation. Despite a bulk of research, so far, many blind spots persist in our understanding of this aspect.

Reliant on written language processing, in this paper, I systematically review a number of scientific works tackling the issue of structural ambiguity in human languages to address the questions, elaborated in the section 2 . The method and data retrieval and cleaning strategy are expanded in the section 3. Summarization of the findings is given in section 4 of the paper, followed by the author's analysis, underlined in section 5 . 


\section{Research questions}

1) What are the main ideas presented in the published articles (20052020) on structural ambiguity resolution in language processing?

2) What are the main venues for unveiling research on structural ambiguity resolution in language processing?

\section{Method}

This study takes up a systematic literature review design linked with a purposive sampling technique [Etikan, Musa, and Alkassim, 2016]. To gather the material for this systematic review, I conducted searches on the topic of attention for research articles in Google Scholar, the most prominent academic engine. Google Scholar has become an alternative source for research content recovery and is useful in systematic review research due its wide-ranging grasp [Van Aalst, 2010].

The following key words were typed in a search box: "structural ambiguity resolution", "reading", and their associated terms. The obtained items were then sorted by several criteria. First, they were arranged by publication type - only research papers, reporting on original and empirical studies, were authorized for consideration, whereas other types of works (books, patents, etc.) were removed due to being mostly a theoretical approach to conducting a study. Second, the found items were filtered by the year of publication by adjusting the custom range through typing years of interest, from 2005 to 2020 , in the designated boxes. Third, the papers for consideration needed to be the most relevant to the topic. This was ensured by considering only the first five pages of search results and checking the sort-by-relevance button.

As a result, the present paper accounts for eight studies, which are found to be the most relevant to the topic and corresponding with the criteria. The retrieved research items are reported through the following three divisions serving as a sort of the order of presentation in this paper: (1) research conducted in the first five year period (2005-2009); (2) in the next five year period (2010-2014); and (3) the past five year period (2015-2020). For each research paper, I record the title of the journal, the publication year, the field of study, as well as the affiliation of the author(s).

\section{Results and discussion}

The issue of structural ambiguity in written language has been addressed by a few of multidisciplinary investigations using a variety of approaches. Van 
Gompel, Pickering, Pearson, and Liversedge (2005) set their goal to reproduce the earlier study [Traxler et al., 1998] by an eye tracking while reading experiment. The researchers recruited English native speakers, who were invited to rate the plausibility of ambiguous relative clause sentences on a Likert scale of 1-7, or very implausible to very plausible, respectively. They were asked several comprehension questions afterwards, to which the subjects had to reply with only yes/no. During the experimental trials, their written language processing was recorded by an eye tracking device to record eye movements (fixations, regressions, etc.), thus, tracing their structural processing difficulty. One of the notable results reveals that when the disambiguation is hindered relative to the preliminary point of ambiguity, relative clauses, which are disambiguated in the direction of high or low attachment, are more challenging to process than globally ambiguous relative clause sentences.

From my own perspective, the significance of the study of Van Gompel and associates rests in supplying the language studies with another verification that low attachment is commonly selected, or more effortlessly processed than high attachment. The study lends the support of the premise that processing sentences with global syntactic ambiguity is way faster than processing sentences with local ambiguity, which in its turn does not really vary from temporal resolution of similar sentences but with no ambiguity. Overall, the study adds up our understanding of language in the sense that the global syntactic ambiguity does not really cause specific difficulties, since the understanding of sentences is somewhat predetermined from an early processing stage.

Swets, Desmet, Hambrick, and Ferreira [2007] examined English and Dutch subjects to establish whether particular variations in working memory properties have an effect on choices concerning attaching an ambiguous element in globally ambiguous relative clause sentences. The experiment with English and Dutch parsers indicates that irrespective of linguistic background, when memory capacity is low, such parsers heavily rely on chunking algorithm than those with a high span. At the same time, such high span readers put together the relative clause with the noun phrase and not the complex nominal group.

The aforementioned study is yet another attempt to find out the status of working memory in processing complex structures and also to check the precise algorithms of understanding such structures for particular languages, namely English and German. The distinct implication of the study by Swets et al. is towards the enrichment of our understanding about the factors, which directly influence our language processing. The authors argue for 
the idea that the capacity of working memory is the key mediator in syntactic processing and understanding. Working memory is also found to impact the strategies parsers take while confronting a multitude of tasks on understanding certain language material.

Long and Prat [2008] administered a reading span task to receive the three groups to participate in the two forthcoming experiments with the use of past-tense and past-participle biased structures. These three groups were formed: low span, medium span, and high span readers. One of the major findings from all the experiments suggests that high span parsers process the prepositional phrase in past-tense biased sentences slower than in the case with the past-participle. Overall findings say that irrespective of span, parsers face more difficulty processing sentences that are influenced to the main verb than when they are inclined to the reduced relative understanding. High span readers showed the effect of verb bias early in the sentence, at the prepositional phrase. Medium span and low span readers do not reveal any ambiguity supposition till reaching the main verb later in a sentence.

This paper, in my view, is one of the not many, which emphasizes attention not on the two groups of parsers but three, i.e. high, medium, and low span parsers. This is, perhaps, one of the main advantages of the study, since earlier studies addressed the working memory constraints through recruiting no more than two groups, namely high and low. That is why, those earlier studies were to some extent restricted in their findings because of missing out one more crucial variable. The study operates to remedy this error in group classification and, by that, advances our contemporary understanding of language processing. The paper similarly gives critical implications for L1-L2 instruction and learning. That is, it directs us to make emphasis on frequent exposure to syntactically complex sentences, involving globally and locally ambiguous sentences, which may accelerate processing of similar structures in future.

Pan and Felser [2011] employed an offline questionnaire alongside an on-line self-paced reading task to check whether referential background knowledge anyhow affects preferences in structural ambiguity resolution in non-native sentence processing and to what extent. The authors demonstrated their stimuli to subjects, namely native Chinese learners of English and native English speakers. The outcome of the research was that the former group showed the quantitative processing of critical regions of the sentences was swayed significantly by the referential context. However, the native speakers were guided by the referential background solely in the off-line 
task. Overall, learners of English, while processing written information, are subtle to some information out of sentence. It gives us grounds to believe that there are capacity limitations for non-native written language processing, irrespective of language proficiency.

The significance of the above-reported study is that it informs language pedagogy about the usefulness of background and pragmatic prompts in L2 processing of syntactically complex structures, rather than for native speakers. That is, in the example of Chinese learners of English as a foreign language, the study augments our awareness about L2 processing, so it is driven by top down material, i. e. contextual information. This study is also to approve the Shallow Structure hypothesis, which puts forward that different kinds of non-grammatical information, among which is background knowledge, are profoundly reliant upon L2 processing.

DeDe [2013] scrutinized the way verb bias affects processing written language in an example of temporarily ambiguous and non-ambiguous sentences by using the same design as Pan and Felser [2011]. A specially designed task featured in a computer program was utilized to show controlled visual stimuli and interact with subjects diagnosed with aphasia. The program displayed the sentences for reading in a self-paced window and in an online fashion, and at the end of the reading task, the participants' job was to answer yes/ no to audially and visually given questions checking their comprehension. The study found that reading times were influenced more due to verb bias and not because of complementizers, while the control group showed that reading times were impacted by the occurrence/non-occurrence of complementisers. It means that there is a disparity among verb bias and sentence structure, which impacts reading non-ambiguous and temporarily ambiguous sentences in aphasia patients.

So, DeDe's study informs language pathology by giving certain insights about aphasiac language processing. She particularly argues in the study that those who are diagnosed with aphasia exhibit better processing of sentences, which fit the lexical preferences of the words when contrasted with structures inconsistent with the argument structure. Her study also affords some suggestions to psycho- and clinical linguistics in that processing times in aphasiac parsers are shorter when verbs were in the syntactic condition, which matched their bias than when they were not. The study may also be valuable for considering procedural aspects of auditory processing mixed with written language processing. 
Acheson and Hagoort [2013] employed the transcranial magnetic stimulation (TMS) to test the demand in the middle temporal gyrus (MTG) and inferior frontal gyrus (IFG) in written language processing and their temporal resolution of engagement in the process of structural disambiguation. The two groups of Dutch speakers, TMS and control, were invited to participate in the neuroimaging study. The experimental stimuli for the study were the structures comprising a temporarily word-class ambiguous word, which might be disambiguated like a noun or a verb reliant on the stimulus background. While the TMS group was processing the sentences, their eye movements were recorded and brain scanned, and the control did not have any brain imaging interpolations. The findings of the mixed approach revealed that parsers are sensitive to the structural ambiguity, which is supported by the elongated reading time data and more fixations for ambiguous parts of structures. The authors got verification for a significant part the IFG obtains when processing ambiguity and no part of the MTG.

Despite the fact that neuroimaging studies are technically challenging, and their results are generally tricky to interpret, the study manages to provide state-of-the-art data with regard to the role of MTG, which was previously undetermined. Also, the study supplements neurolinguistics with some new evidence about IFG. In particular, MTG is set off substantially not only when we see faces or estimate distance, but also when we gain access to word meaning while written language processing, as well as when confronted with plausible and implausible conditions. The left part of the IFG is found to be crucial in regulating context sensitivity and semantic processing while reading, in addition to the known role in picture naming.

Martin and McElree [2018] studied American English native speakers while the latter were processing structurally ambiguous and unambiguous structures. Once the subjects found a particular sentence structurally ambiguous, they had to respond with yes/no by pressing designated buttons. "Yes" meant that a sentence was non-ambiguous, while "No" indicated that there was doubt regarding sentence clarity. It was discovered that ambiguous structures' speed-accuracy tradeoff (SAT) times were elongated as compared to non-ambiguous sentences. Retrieval cues, more strongly related to the true subject, always increase accuracy, regardless of ambiguity. These findings are consistent with a language processing architecture where cue-driven operations give rise to analysis, and wherein diagnostic cues support retrieval, regardless of parsing difficulty or structural uncertainty. 
This study provides another account for that quantitative data delivers resources for exploring general structural issues in language processing. For example, whether there are unforeseen consequences in the organization of operations in structural units, with certain tasks having temporal significance more than others, against arranged in an interactive way. Martin and McElree add up with their study to our understanding of language processing concerning erroneous recovery from memory, such as some structurally inappropriate items, can prompt a reanalysis of structures by applying continual recovery efforts.

Stella and Engelhardt [2019] tested subjects with dyslexia and healthy subjects by eye tracker while both groups were processing temporary structural ambiguities. The goal was to check whether those with dyslexia face complications while processing temporary structurally ambiguous subordinate-main sentences as well as unambiguous sentences. As it might be projected, subjects with dyslexia failed to process written language material better than healthy subjects and indicated slower processing of the disambiguating region while reading structurally ambiguous sentences.

To the best of my knowledge, this paper appears to be the only one to aim at an understudied groups, namely adolescent and college students, tested for literacy skills and working memory limitations in dyslexic parsers. Therefore, it informs language pathology generally about how parsers with dyslexia progress throughout written language at the sentence level. It also stimulates to shape the special teaching methods to be employed while working with dyslexic language learners. Additionally, the study reinforces the idea how both online and offline methods may coordinate our knowledge of syntactic disambiguation.

The examined papers are available from the following venues (Table 1). It must be noted all the papers are published in peer-reviewed journals indexed by reputable science databases, such as Web of Science, Scopus, ERIH PLUS, etc. These journals are at the crossroads of 12 fields of study and research areas: psycholinguistics, experimental psychology, cognitive science, neuroscience, neuropsychology, neurobiology, general linguistics, computer science, philosophy, language pathology, neurology, and neurolinguistics.

\section{Conclusions}

The reported studies make an attempt to deliver universal inferences about syntactic disambiguation in language processing through conducting 


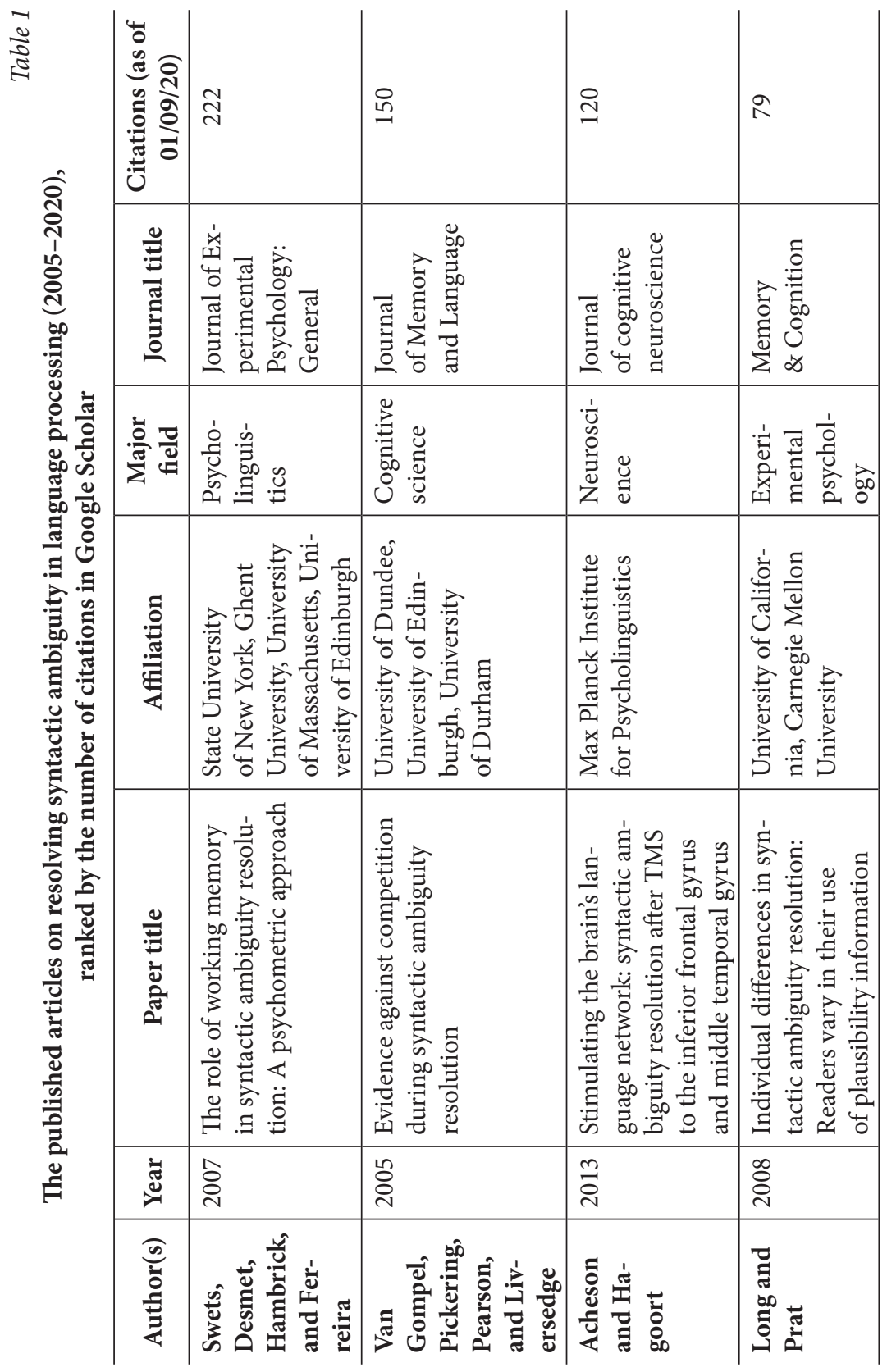




\begin{tabular}{|c|c|c|c|c|}
\hline 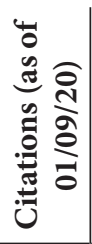 & in & $=$ & $a$ & $m$ \\
\hline 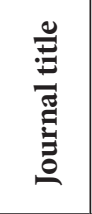 & $\begin{array}{l}\text { 㺃 } \\
\text { 永 }\end{array}$ & 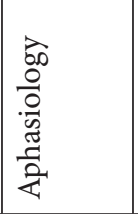 & 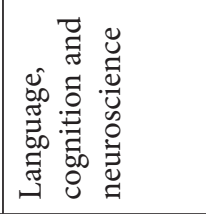 & 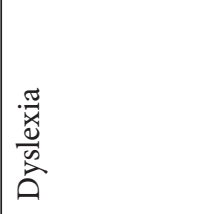 \\
\hline$\stackrel{\frac{0}{\pi}}{\frac{\pi}{2}}$ & 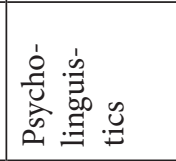 & 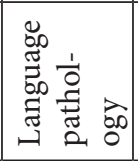 & 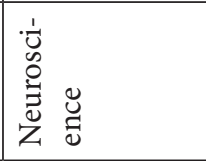 & 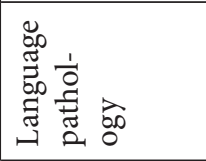 \\
\hline 莺 & 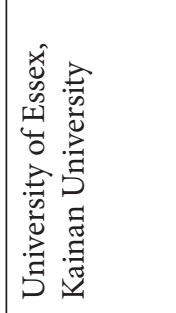 & 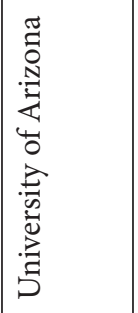 & 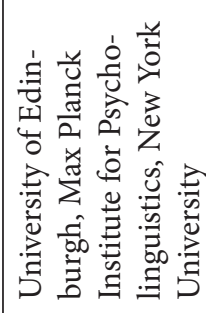 & 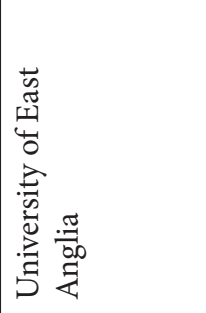 \\
\hline 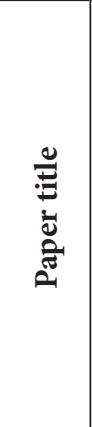 & 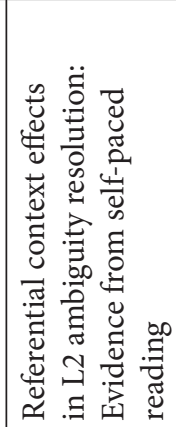 & 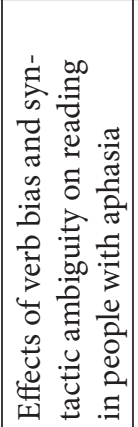 & 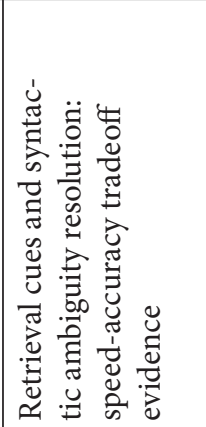 & 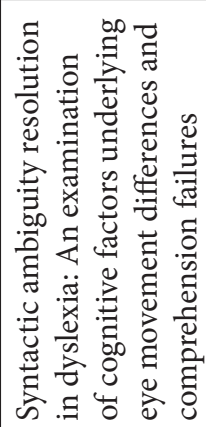 \\
\hline 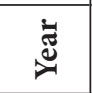 & $\overrightarrow{\vec{i}}$ & $\mid \vec{n}$ & $\stackrel{\infty}{\stackrel{\sim}{\sim}}$ & $\overrightarrow{\vec{i}}$ \\
\hline 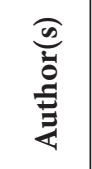 & 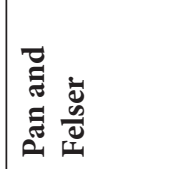 & อั๊ & 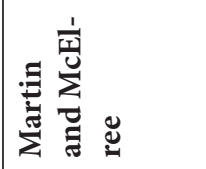 & 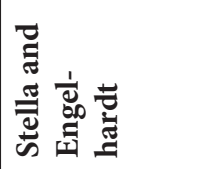 \\
\hline
\end{tabular}


experimental studies with the use of neuroimaging techniques, eye-tracking devices, and psychophysical tests.

The latter are found to be more prevalent since they do not typically involve any further convolutions and technical issues at the stage of interpreting the results. I also explain this occurrence by inevitable challenges at the onset of studies when recruiting participants. Trials, which involve additional equipment as an electromagnetic coil as in TMS or an eye-tracker device in eye-tracking experiments, are usually averted because of various reasons. One of them is that some potential participants are worried about the negative impact of those tools on their health system. This may happen regardless of informing potential participants about no potential harm or damage concealed in employing those non-invasive devices.

The polarity of findings on syntactic disambiguation leaves room for much to be discovered. What is already recognized in the literature of 20052020 is that the capacity of working memory and the degree of span in parsers have a considerable influence on processing complex structures and specially structurally ambiguous sentences. In fact, global structural ambiguities are processed faster than local ambiguities. In processing, working memory is a catalyst for forming reading strategies for different sentence types.

I believe more research will arrive to elaborate on syntactic disambiguation in language processing and explain other aspects. In this regard, more reviews need to be conducted for critical appraisal and our further understanding. In conclusion, I suggest forthcoming systematic reviews to be broader and to report, for instance, on studies of the past 30-50 years about resolving syntactic ambiguity in language processing, and those searchers would have to include additional key words to mitigate the risk of misleading outcomes.

\section{Acknowledgment}

The author extends her gratitude to Dr. Luk Pei Sui Zoe and Dr. Lee Kwing Lok Albert for their motivating inspiration throughout conducting the systematic review.

\section{References:}

1. D. J. Acheson, P. Hagoort. Stimulating the brain's language network: syntactic ambiguity resolution after TMS to the inferior frontal gyrus and middle temporal gyrus. Journal of cognitive neuroscience, No. 25 (10) (2013), pp. 1664-1677.

2. L. Darzhinova. Processing of written sentences on the example of Russian-English bilinguals. Indonesian Research Journal in Education, No. 3(2) (2019), pp. 421-433. 
3. G. DeDe. Effects of verb bias and syntactic ambiguity on reading in people with aphasia. Aphasiology, No. 27(12) (2013), pp. 1408-1425.

4. P.E. Dussias. Uses of eye-tracking data in second language sentence processing research. Annual Review of Applied Linguistics, No. 30 (2010), p. 149.

5. I. Etikan, S. A. Musa, R.S. Alkassim. Comparison of convenience sampling and purposive sampling. American journal of theoretical and applied statistics, No. 5 (1) (2016), pp. 1-4.

6. E. Kaan, J. Kirkham, F. Wijnen. Prediction and integration in native and second-language processing of elliptical structures. Bilingualism, No. 19(1) (2016), p. 1.

7. J.H. Lim, K. Christianson. Second language sentence processing in reading for comprehension and translation. Bilingualism, No. 16(3) (2013), p. 518.

8. D. L. Long, C. S. Prat. Individual differences in syntactic ambiguity resolution: Readers vary in their use of plausibility information. Memory \& Cognition, No. 36(2) (2008), pp. 375-391.

9. A.E. Martin, B. McElree. Retrieval cues and syntactic ambiguity resolution: speed-accuracy tradeoff evidence. Language, cognition and neuroscience, No. 33(6) (2018), pp. 769-783.

10. H. Y. Pan, C. Felser. Referential context effects in L2 ambiguity resolution: Evidence from self-paced reading. Lingua, No. 121(2) (2011), pp. 221-236.

11. M. Stella, P.E. Engelhardt. Syntactic ambiguity resolution in dyslexia: An examination of cognitive factors underlying eye movement differences and comprehension failures. Dyslexia, No. 25(2) (2019), pp. 115-141.

12. B. Swets, T. Desmet, D.Z. Hambrick, F. Ferreira. The role of working memory in syntactic ambiguity resolution: A psychometric approach. Journal of Experimental Psychology: General, No. 136(1) (2007), p. 64.

13. M. J. Traxler, M. J. Pickering, C. Jr. Clifton. Adjunct attachment is not a form of lexical ambiguity resolution. Journal of Memory and Language, No. 39(4) (1998), pp. 558-592.

14. J. Van Aalst. Using Google Scholar to estimate the impact of journal articles in education. Educational researcher, No. 39(5) (2010), pp. 387-400.

15. R. P. Van Gompel, M. J. Pickering, J. Pearson, S. P. Liversedge. Evidence against competition during syntactic ambiguity resolution. Journal of Memory and Language, No. 52(2) (2005), pp. 284-307. 